Quantum Information and Computation, Vol. 0, No. 0 (2003) 000-000

(C) Rinton Press

\title{
Mathematical framework for detection and quantification of nonclassical correlation
}

\author{
AKIRA SAITOH ${ }^{1}$, ROBABEH RAHIMI ${ }^{1,2}$, MIKIO NAKAHARA ${ }^{1,3}$ \\ ${ }^{1}$ Research Center for Quantum Computing, Interdisciplinary \\ Graduate School of Science and Engineering, Kinki University \\ 3-4-1 Kowakae, Higashi-Osaka, Osaka 577-8502, Japan \\ ${ }^{2}$ Department of Chemistry and Materials Science \\ Graduate School of Science, Osaka City University \\ 3-3-138 Sugimoto, Sumiyoshi-ku, Osaka 558-8585, Japan \\ ${ }^{3}$ Department of Physics, Kinki University \\ 3-4-1 Kowakae, Higashi-Osaka, Osaka 577-8502, Japan
}

Received (received date)

Revised (revised date)

\begin{abstract}
Existing measures of bipartite nonclassical correlation that is typically characterized by nonvanishing nonlocalizable information under the zero-way CLOCC protocol are expensive in computational cost. We define and evaluate economical measures on the basis of a new class of maps, eigenvalue-preserving-but-not-completely-eigenvalue-preserving (EnCE) maps. The class is in analogy to the class of positive-but-not-completely-positive (PnCP) maps that have been commonly used in the entanglement theories. Linear and nonlinear EnCE maps are investigated. We also prove subadditivity of the measures in a form of logarithmic fidelity.
\end{abstract}

Keywords: Nonclassical correlation, Subadditive measures

Communicated by: to be filled by the Editorial

\section{Introduction}

Nonclassical correlation of a bipartite system is an essential resource to perform quantum information processing [1, 2]. Entanglement, namely the degree of inseparability, of a system is the most well-known nonclassical correlation. Besides the entanglement paradigm, several different paradigms [3, 4, 5] have been proposed in which the set of the states with nonclassical correlation includes certain nonentangled states. Unlike entangled states that are defined on the basis of the impossibility of preparation under local operations and classical communications (LOCC) [6], nonclassically correlated states in the different paradigms have been defined on the basis of post-preparation stages.

Historically, nonlocality about locally nonmeasurable separable states was discussed by Bennett et al. 3. Later, in the context of system-apparatus correlation, a measure called quantum discord was defined by Ollivier and Zurek 4, which is a discrepancy of two expressions of a mutual information that are equivalent in the regime of classical information theory. Recently, the term quantum discord often indicates the minimized one over the possible local (orthogonal) projection sets and is widely used as a measure of nonclassical correlation. As a 
typical example that justifies the computational power of nonclassical correlation other than entanglement, it was reported by Datta et al. 7] that the Knill and Laflamme's fast estimation of a normalized trace of a unitary matrix, which uses a single pseudo-pure qubit and the remaining qubits in a maximally mixed state [8], exhibits a large quantum discord and vanishing entanglement. Another well-known definition of nonclassical correlation is the quantum deficit proposed by Oppenheim et al. [5], which is nonlocalizable information under the closed LOCC (CLOCC) protocol that allows only local unitary operations and operations to send subsystems through a complete dephasing channel. Among different setups they considered [9], the zero-way setting - namely a setting in which the players are allowed to communicate under CLOCC only after local complete dephasing possibly subsequent to local unitary operations - was connected to a mathematically simple classical/nonclassical separation.

The quantum deficit for a density matrix $\rho^{\mathrm{AB}}$ of a bipartite system $\mathrm{AB}$ is defined as 9 ]

$$
\min _{\Lambda \in \mathrm{CLOCC}}\left[S_{\mathrm{vN}}\left(\operatorname{Tr}_{\mathrm{B}} \Lambda \rho^{\mathrm{AB}}\right)+S_{\mathrm{vN}}\left(\operatorname{Tr}_{\mathrm{A}} \Lambda \rho^{\mathrm{AB}}\right)\right]-S_{\mathrm{vN}}\left(\rho^{\mathrm{AB}}\right),
$$

where $S_{\mathrm{vN}}(\cdot)$ is the von Neumann entropy. Here, the system may be kept by a single player after the process $\Lambda$, i.e., $\operatorname{Tr}_{\mathrm{B}} \Lambda \rho^{\mathrm{AB}}$ or $\operatorname{Tr}_{\mathrm{A}} \Lambda \rho^{\mathrm{AB}}$ possibly becomes a null state. The entropy of a null state is defined to be zero. In case of the zero-way setting, the minimum is obtained when a single player possesses the total system after the process. Thus the zero-way quantum deficit is equal to a minimum discrepancy between $S_{\mathrm{vN}}\left[\left(\mathcal{V}^{\mathrm{A}} \otimes \mathcal{V}^{\mathrm{B}}\right) \rho^{\mathrm{AB}}\right]$ and $S_{\mathrm{vN}}\left(\rho^{\mathrm{AB}}\right)$ where $\mathcal{V}^{\mathrm{A}, \mathrm{B}}$ is a dephasing operation (acting on a local system) deleting the off-diagonal elements of a target matrix on a certain local basis; the minimum is taken over all local bases.

The zero-way quantum deficit vanishes if the state has a product eigenbasis. A quantum bipartite system is called (properly) classically correlated [9] if and only if it is described by a density matrix having a product eigenbasis (PE),

$$
\rho_{\mathrm{PE}}^{\mathrm{AB}}=\sum_{j, k=1,1}^{d^{\mathrm{A}}, d^{\mathrm{B}}} e_{j k}\left|v_{j}^{\mathrm{A}}\right\rangle\left\langle v_{j}^{\mathrm{A}}|\otimes| v_{k}^{\mathrm{B}}\right\rangle\left\langle v_{k}^{\mathrm{B}}\right|,
$$

where $d^{\mathrm{A}}\left(d^{\mathrm{B}}\right)$ is the dimension of the Hilbert space of $\mathrm{A}(\mathrm{B}), e_{j k}$ is the eigenvalue of $\rho$ corresponding to the eigenvector $\left|v_{j}^{\mathrm{A}}\right\rangle \otimes\left|v_{k}^{\mathrm{B}}\right\rangle$. Thus, a quantum bipartite system consisting of subsystems A and B is nonclassically correlated if and only if it is described by a density matrix having no product eigenbasis.

Using this simple classical/nonclassical separation, other measures [10, 11] were later proposed. In particular, Piani et al. [12] recently designed a measure which vanishes if and only if a state has a product eigenbasis. It is in a similar form as the quantum discord [4 and defined as a distance of two different quantum mutual informations that is minimized over local maps associated with local positive operator-valued measurements [13. It has also been known [4] that the quantum discord vanishes for both of the system-apparatus and apparatus-system settings if and only if a state has a product eigenbasis.

A pending problem is that the original nonlocalizable information and the Piani et al.'s measure both require expensive computational tasks to take minimums over all possible local operations. A similar difficulty exists in Groisman et al.'s measure [10 which is a discrepancy between an original state $\rho^{\mathrm{AB}}$ and the state after dephasing under an eigenbasis of $\operatorname{Tr}_{\mathrm{B}} \rho^{\mathrm{AB}} \otimes$ $\operatorname{Tr}_{\mathrm{A}} \rho^{\mathrm{AB}}$ (called Schmidt basis). In fact, the obviously classically correlated state $(|00\rangle\langle 00|+$ 
$|11\rangle\langle 11|) / 2$ is mapped to $I / 4$ by dephasing when the improper Schmidt basis $\{|0\rangle,|1\rangle\} \otimes\{| \pm\rangle\}$ with $| \pm\rangle=(|0\rangle \pm|1\rangle) / \sqrt{2}$ is chosen while it is mapped to itself when a proper Schmidt basis (the computational basis in this case) is chosen. Thus a minimization over possible Schmidt bases is required. The recently-proposed measurement-induced disturbance measure [14, 15] is a variant of the Groisman et al.'s measure; the same problem exists. In general, the measures involving a minimization over local operations are intractable in view of computational cost.

In our previous contribution [11, an entropic measure $G$ based on a sort of game to find the eigenvalues of a reduced density matrix from the eigenvalues of an original density matrix was proposed, in the context of $m$-partite $m$-split nonclassical correlation. This measure can be computed within a finite time although it does not have a perfect detection range. Its computational cost is indeed less than those for the intractable measures, but still exponential in the dimension of the Hilbert space.

Here we introduce a new class of maps to define measures with improved computational cost, in the context of bipartite splitting. It is the class of eigenvalue-preserving-but-notcompletely-eigenvalue-preserving (EnCE) maps. We find it analogous to the class of positivebut-not-completely-positive (PnCP) maps [16, 17, 18, that are popularly used for detection and quantification of entanglement. The idea of introducing the class EnCE was briefly mentioned in our previous contribution [19]. Here, we give mathematically strict definitions and show the fact that any linear EnCE map is a concatenation of unitary and anti-unitary operations. Thus the restriction of the theory is clarified. We further introduce a measure using a nonlinear EnCE map in order for achieving a wider detection range.

The measures we propose here on the basis of EnCE maps are not as strong as those using an infinite number of trials, in the detection range of nonclassically correlated states. We propose a simple way to relax this drawback: The detection range is improved by introducing an average of multiple measures whose detection ranges are mutually different. This approach is described in Section 4.4 .

This paper is organized as follows. We start with an overview of the conventional theory of PnCP maps in Section 2. We then define and evaluate new classes of maps in Section 3 The measures are defined and their properties, such as subadditivity, are verified in Section 4. First non-subadditive measures are introduced in Section 4.1. Second subadditive measures are introduced in Section 4.2 with the proof of the subadditivity. The computational complexity of the subadditive measures is investigated in Section 4.3. A simple way to relax the drawback in the detection range of the measures is shown in Section 4.4. Section 5 summarizes our results with some remarks.

\section{Conventional theory of PnCP maps}

Quantum physics is governed by completely positive (CP) maps. Any map which is not CP $(\mathrm{nCP})$ is considered to be physically unfeasible. There is, however, a class of nCP maps which are useful for characterizing entanglement. These maps are in the class of positive-but-notcompletely-positive (PnCP) maps. It has been more than a decade since the Peres-Horodecki criterion opened the mathematical study of PnCP maps [16, 17, 18. A PnCP map $\Lambda_{\mathrm{PnCP}}$ is positive when acting as a global operator but nonpositive when acting as $I \otimes \Lambda_{\mathrm{PnCP}}$ on a system. It maps a separable state $\rho_{\text {sep }}=\sum_{i} w_{i} \rho_{i}^{\mathrm{A}} \otimes \rho_{i}^{\mathrm{B}}$ of a bipartite system AB with nonnegative weights $w_{i}$ to a certain (physically feasible) state, while it does not necessarily 
map an inseparable state to a positive Hermitian matrix. Thus one finds a density matrix inseparable if one detects a negative eigenvalue of the matrix obtained after applying $I \otimes \Lambda_{\mathrm{PnCP}}$ to the density matrix. The PnCP map theory has gathered a broad interest in relation to detecting entanglement (See, e.g., Ref. [20]).

One might be curious to find an analogue of the PnCP map theory to detect nonclassical correlations often defined in different ways [3, 4, 5] than that of entanglement. We pursue the analogous theory to detect nonclassical correlation in the context of classical/nonclassical separation given by the existence/absence of a product eigenbasis of a bipartite state.

\section{Introduction of unconventional classes of maps and their use}

We aim to introduce an analogy of the PnCP map theory to the present paradigm of classical/nonclassical separation. For this purpose, we define our new classes of maps. Let us start with a linear map theory.

Definition 1 An eigenvalue-preserving (EP) map $\Lambda_{\mathrm{EP}}$ is a map acting on a general $d \times d$ density matrix $\rho=\sum_{k=1}^{d} e_{k}\left|v_{k}\right\rangle\left\langle v_{k}\right|$ (here, $e_{k}$ and $\left|v_{k}\right\rangle\left\langle v_{k}\right|$ are the $k$ th eigenvalue and the corresponding projector, respectively) such that

$$
\rho=\sum_{k=1}^{d} e_{k}\left|v_{k}\right\rangle\left\langle v_{k}\left|\stackrel{\Lambda_{\mathrm{EP}}}{\mapsto} \rho^{\prime}=\sum_{k=1}^{d} e_{k}\right| v_{k}^{\prime}\right\rangle\left\langle v_{k}^{\prime}\right|
$$

where $\left\{\left|v_{k}\right\rangle\right\}_{k}$ and $\left\{\left|v^{\prime}{ }_{k}\right\rangle\right\}_{k}$ are both complete orthonormal systems (CONSs). The dimension of $\rho^{\prime}$ is equal to $d$.

Alternatively, we may define the EP map in the following way:

Definition 2 An EP map $\Lambda_{\mathrm{EP}}$ acting on a quantum system $S$ is a bijection between the set of projectors $\left\{\left|v_{k}\right\rangle\left\langle v_{k}\right|\right\}_{k=1}^{d}$ generated from the vectors $\left|v_{k}\right\rangle$ of a CONS to the set of projectors $\left\{\left|v^{\prime}{ }_{k}\right\rangle\left\langle v^{\prime}{ }_{k}\right|\right\}_{k=1}^{d}$ generated from the vectors $\left|v^{\prime}{ }_{k}\right\rangle$ of a CONS for any CONS $\left\{\left|v_{k}\right\rangle\right\}_{k=1}^{d}$ of the Hilbert space of $S$.

A class of EP maps analogous to $\mathrm{CP}$ is defined as follows.

Definition 3 An EP map $\Lambda$ is a complete EP (CEP) map if and only if $I \otimes \Lambda$ is also an EP map for identity map I of arbitrary dimension. We denote such $\Lambda$ as $\Lambda_{\mathrm{CEP}}$.

Observation 1 One of the simplest CEP maps is $\widetilde{\mathcal{U}}(d): \rho \rightarrow \tilde{u} \rho \tilde{u}^{\dagger}$ where $\tilde{u}$ is an element of a flag manifold $\widetilde{\mathrm{U}}(d)=\mathrm{U}(d) / \mathrm{U}(1)^{\times d}$ (here, $\mathrm{U}(d)$ is the $d$-dimensional unitary group).

We now define the PnCP analogy in the following way.

Definition 4 An EP map $\Lambda$ is an EP-but-not-completely-EP (EnCE) map if and only if there exists an identity map $I$ of some dimension, for which $I \otimes \Lambda$ is not an EP map. We denote such $\Lambda$ as $\Lambda_{\mathrm{EnCE}}$.

We have defined a class of EnCE maps. As is analogous to the usage of a PnCP map, the usage of a linear EnCE map is to find a certain change of eigenvalues of a density matrix by applying the map to a local subsystem. This is based on the following proposition.

Proposition 1 Both $I^{\mathrm{A}} \otimes \Lambda_{\mathrm{EnCE}}^{\mathrm{B}}$ and $\Lambda_{\mathrm{EnCE}}^{\mathrm{A}} \otimes I^{\mathrm{B}}$ preserve the eigenvalues of a density matrix of a system $\mathrm{AB}$ if the density matrix has a product eigenbasis. 
Proof. Let the density matrix with a product eigenbasis $\left\{\left|v_{j}^{\mathrm{A}}\right\rangle\left|v_{k}^{\mathrm{B}}\right\rangle\right\}_{j, k=1,1}^{d^{\mathrm{A}}, d^{\mathrm{B}}}$ be

$$
\sigma^{\mathrm{AB}}=\sum_{j, k=1,1}^{d^{\mathrm{A}}, d^{\mathrm{B}}} e_{j k}\left|v_{j}^{\mathrm{A}}\right\rangle\left\langle v_{j}^{\mathrm{A}}|\otimes| v_{k}^{\mathrm{B}}\right\rangle\left\langle v_{k}^{\mathrm{B}}\right|,
$$

where $e_{j k}$ is the $(j k)$ th eigenvalue corresponding to the $(j k)$ th projector $\left|v_{j}^{\mathrm{A}}\right\rangle\left\langle v_{j}^{\mathrm{A}}|\otimes| v_{k}^{\mathrm{B}}\right\rangle\left\langle v_{k}^{\mathrm{B}}\right|$. By the definition, any linear EP map acting as a local operation should map an eigenbasis of the reduced density matrix of a target subsystem to another CONS. Therefore, it is obvious that

$$
\left(I^{\mathrm{A}} \otimes \Lambda_{\mathrm{EnCE}}^{\mathrm{B}}\right) \sigma^{\mathrm{AB}}=\sum_{j, k=1,1}^{d^{\mathrm{A}}, d^{\mathrm{B}}} e_{j k}\left|v_{j}^{\mathrm{A}}\right\rangle\left\langle v_{j}^{\mathrm{A}}|\otimes|{v^{\prime}}_{k}^{\mathrm{B}}\right\rangle\left\langle{v^{\prime}}_{k}^{\mathrm{B}}\right|,
$$

where $\left\{\left|v_{k}^{\prime}{ }_{k}^{\mathrm{B}}\right\rangle\right\}_{k}$ is a CONS of the Hilbert space of B, which may be different from $\left\{\left|v_{k}^{\mathrm{B}}\right\rangle\right\}_{k}$. It is trivial to show the same proof applies to $\Lambda_{\mathrm{EnCE}}^{\mathrm{A}} \otimes I^{\mathrm{B}}$.

Corollary 1 A density matrix $\rho^{\mathrm{AB}}$ has no product eigenbasis if either $\left(I^{\mathrm{A}} \otimes \Lambda_{\mathrm{EnCE}}^{\mathrm{B}}\right) \rho^{\mathrm{AB}}$ or $\left(\Lambda_{\mathrm{EnCE}}^{\mathrm{A}} \otimes I^{\mathrm{B}}\right) \rho^{\mathrm{AB}}$ has eigenvalues different from those of $\rho^{\mathrm{AB}}$.

Proof. This is the contraposition of Proposition $1, \quad \square$.

There is, however, a restriction in the type of the linear EnCE maps according to the following proposition. This restriction is later relaxed by a nonlinear EnCE map.

Proposition 2 Any linear EP map can be decomposed into unitary transformations and a transposition. Hence any linear EnCE map can be decomposed into unitary transformations and a transposition.

Proof. Consider a linear EP map $\Lambda_{\text {lin }}$ and two pure states $|x\rangle\langle x|$ and $|y\rangle\langle y|(|x\rangle$ and $|y\rangle$ can be nonorthogonal to each other). Let $\left|x^{\prime}\right\rangle\left\langle x^{\prime}\right|=\Lambda_{\operatorname{lin}}(|x\rangle\langle x|)$ and $\left|y^{\prime}\right\rangle\left\langle y^{\prime}\right|=\Lambda_{\operatorname{lin}}(|y\rangle\langle y|)$. Consider a state $\tau=|x\rangle\langle x|+| y\rangle\langle y|$ represented under a certain CONS. As $\Lambda_{\text {lin }}$ changes this CONS to a certain CONS, $\langle x|\tau| x\rangle$ is equal to $\left\langle x^{\prime}\left|\Lambda_{\operatorname{lin}}(\tau)\right| x^{\prime}\right\rangle$. This suggests that $|\langle x \mid y\rangle|=\left|\left\langle x^{\prime} \mid y^{\prime}\right\rangle\right|$ since $\Lambda_{\text {lin }}$ is a linear map. Note that this is true for any linear EP map $\Lambda_{\text {lin }}$ and any two pure states $|x\rangle$ and $|y\rangle$. Therefore, by Wigner's unitary-antiunitary theorem [21], there are only two possible types for $\Lambda_{\text {lin }}$, namely, unitary and antiunitary 22] transformations acting on a target density matrix. Hence the proposition holds.

Observation 2 A unique nontrivial linear EnCE map is the transposition $\Lambda_{\mathrm{T}}$, according to the above proposition. As an example of detecting a nonclassical correlation, consider the density matrix of a two-qubit pseudo-entangled (PS) state,

$$
\rho_{\mathrm{ps}}=(1-p) I / 4+p|\psi\rangle\langle\psi|
$$

with $|\psi\rangle=(|00\rangle+|11\rangle) / \sqrt{2}$ and $0<p \leq 1$. It has a nondegenerate eigenvalue $(1+3 p) / 4$ and a degenerate eigenvalue $(1-p) / 4$ with multiplicity 3 . Its partial transposition, $\left(I \otimes \Lambda_{\mathrm{T}}\right) \rho_{\mathrm{ps}}$, has a nondegenerate eigenvalue $(1-3 p) / 4$ and a degenerate eigenvalue $(1+p) / 4$ with multiplicity 3 . These two sets of eigenvalues are different for $p>0$, indicating the existence of a nonclassical correlation.

It should be noted that having different eigenvalues after partial transposition is a sufficient but not necessary condition for a state to have no product eigenbasis. For example, a 2-qubit state

$$
\rho_{0+}=\frac{1}{2}(|00\rangle\langle 00|+|++\rangle\langle++|)
$$


with $|+\rangle=(|0\rangle+|1\rangle) / \sqrt{2}$ has no product eigenbasis because $|0\rangle\langle 0|$ and $|+\rangle\langle+|$ cannot be diagonalized simultaneously. It is clear that the partial transposition does not change the state and hence it does not detect a nonclassical correlation. We may use, instead, the nonlinear map $\mathcal{P}_{x}$ defined later in order to detect a nonclassical correlation of this state.

The use of a linear EnCE map for detecting nonclassical correlation is intuitive and technically easy as we have seen. There is, however, a case where the limitation of the linear EnCE maps is clearly observed as described below.

Remark 1 There are states called one-way classically correlated (1WCC) states [9], in the form

$$
\rho_{1 \mathrm{WCC}}=\sum_{i}\left|i^{\mathrm{x}}\right\rangle\left\langle i^{\mathrm{x}}\right| \otimes \sigma_{i}^{\mathrm{y}}
$$

with $\left|i^{\mathrm{x}}\right\rangle$ a CONS of $\mathrm{x}=\mathrm{A}$ or $\mathrm{B}$ and $\mathrm{y}$ the remaining system; $\sigma_{i}^{\mathrm{y}}$ (unnormalized) density operators acting on $\mathrm{y}$, dependent on the index $i$. Such a state may have no product eigenbasis but testing a change in the eigenvalues under $I^{\mathrm{x}} \otimes \Lambda_{\mathrm{EnCE}}^{\mathrm{y}}$ for a single side is not enough to detect it. Therefore we need to test for both $(\mathrm{x}, \mathrm{y})=(\mathrm{A}, \mathrm{B})$ and $(\mathrm{x}, \mathrm{y})=(\mathrm{B}, \mathrm{A})$.

Proposition 3 One cannot detect a nonclassical correlation of a one-way classically correlated $(1 \mathrm{WCC})$ state using a linear EnCE map.

Proof. It is easy to find that applying a partial transposition to $\rho_{1 \mathrm{WCC}}$ results in either $\left(U_{*}^{\mathrm{x}} \otimes I^{\mathrm{y}}\right) \rho_{1 \mathrm{WCC}}\left(U_{*}^{\mathrm{x}} \otimes I^{\mathrm{y}}\right)^{\dagger}$ or $\left(U_{*}^{\mathrm{x}} \otimes I^{\mathrm{y}}\right)^{\dagger} \rho_{1 \mathrm{WCC}}^{*}\left(U_{*}^{\mathrm{x}} \otimes I^{\mathrm{y}}\right)$ with $U_{*}^{\mathrm{x}}=\sum_{i}\left(\left|i^{\mathrm{x}}\right\rangle^{*}\right)\left\langle i^{\mathrm{x}}\right|$. In addition, any partial unitary transformation preserves the eigenvalues of $\rho_{1 \mathrm{WCC}}$. By Proposition 2 , the proof is completed.

This proposition suggests that we need to search for nonlinear EnCE maps for a wider range of detection than that of linear ones. The definition involving both linear and nonlinear ones should be newly given in consistent with Proposition 1 .

Definition 5 An EnCE map $\Lambda_{\mathrm{EnCE}}$ (that can be nonlinear) should have the following properties.

(i) For any density matrix $\rho=\sum_{k=1}^{d} e_{k}\left|v_{k}\right\rangle\left\langle v_{k}\right|$,

$\Lambda_{\mathrm{EnCE}}: \rho \mapsto \sum_{k=1}^{d} e_{k}\left|v_{k}{ }^{\prime}\right\rangle\left\langle v_{k}{ }^{\prime}\right|$ where $\left\{\left|v_{k}\right\rangle\right\}$ and $\left\{\left|v_{k}{ }^{\prime}\right\rangle\right\}$ are both CONSs; $e_{k}$ are the eigenvalues ${ }^{a}$

(ii) For any bipartite density matrix $\rho_{\mathrm{PE}}^{\mathrm{AB}}$ with a product eigenbasis, written as (1), $I^{\mathrm{A}} \otimes \Lambda_{\mathrm{EnCE}}^{\mathrm{B}}: \rho_{\mathrm{PE}}^{\mathrm{AB}} \mapsto \widehat{\rho^{\mathrm{AB}}}$ where $\widehat{\rho^{\mathrm{AB}}}$ is an Hermitian matrix with the set of the eigenvalues same as that of $\rho_{\mathrm{PE}}^{\mathrm{AB}}$.

(iii) For some bipartite density matrix $\sigma^{\mathrm{AB}}$ having no product eigenbasis, $I^{\mathrm{A}} \otimes \Lambda_{\mathrm{EnCE}}^{\mathrm{B}}$ maps it to an Hermitian matrix with the set of the eigenvalues different from that of $\sigma^{\mathrm{AB}}$.

We find that there is, in fact, a useful nonlinear EnCE map. To define it, we first introduce the specially-designed nonlinear map $\Gamma_{x}$.

Definition 6 A nonlinear map $\Gamma_{x}$ acting on a (possibly unnormalized) quantum state $\rho$ is defined as follows.

$$
\begin{aligned}
& \Gamma_{x}: \rho \mapsto \sqrt{\left(\rho \rho^{x-1}\right)(\text { h.c. })}=\rho^{x}, \\
& I^{\mathrm{A}} \otimes \Gamma_{x}^{\mathrm{B}}: \rho^{\mathrm{AB}} \mapsto \sqrt{\left\{\rho^{\mathrm{AB}}\left[I^{\mathrm{A}} \otimes\left(\operatorname{Tr}_{\mathrm{A}} \rho^{\mathrm{AB}}\right)^{x-1}\right]\right\}\{\text { h.c. }\}},
\end{aligned}
$$

${ }^{a}$ This is a property of any (possibly nonlinear) EP map. 
where $x \in \mathbf{R}$; the square root is positive and h.c. stands for Hermitian conjugate (conjugate transpose).

[Here, the inverse of a density matrix $\rho=\sum_{k} e_{k}\left|v_{k}\right\rangle\left\langle v_{k}\right|$, with $\left(e_{k},\left|v_{k}\right\rangle\right)$ the pair of an eigenvalue and the (normalized) corresponding eigenvector, is defined as $\left.\rho^{-1} \equiv \sum_{k, c_{k} \neq 0} c_{k}^{-1}\left|v_{k}\right\rangle\left\langle v_{k}\right|.\right]$ This is an extension of the $x$ th power of a matrix. Note that $\left(I^{\mathrm{A}} \otimes \Gamma_{x}^{\mathrm{B}}\right) \rho^{\mathrm{AB}}$ is a quantum state (positive Hermitian matrix) because, for positive Hermitian matrices $A$ and $B,(A B)$ (h.c.) $=$ $A B B A$ is a positive Hermitian matrix.

A nonlinear EnCE map is now defined by using $\Gamma_{x}$.

Definition 7 A nonlinear EnCE map $\mathcal{P}_{x}$ acting on a quantum state $\rho$ is defined as follows.

$$
\begin{aligned}
& \mathcal{P}_{x}: \rho^{\mathrm{AB}} \mapsto \Gamma_{1 / x} \Gamma_{x} \rho^{\mathrm{AB}}=\rho^{\mathrm{AB}}, \\
& I^{\mathrm{A}} \otimes \mathcal{P}_{x}^{\mathrm{B}}: \rho^{\mathrm{AB}} \mapsto\left(I^{\mathrm{A}} \otimes \Gamma_{1 / x}^{\mathrm{B}}\right)\left(I^{\mathrm{A}} \otimes \Gamma_{x}^{\mathrm{B}}\right) \rho^{\mathrm{AB}},
\end{aligned}
$$

where $x \in \mathbf{R}, x \neq 1$.

Of course, $\left(I^{\mathrm{A}} \otimes \mathcal{P}_{x}^{\mathrm{B}}\right) \rho^{\mathrm{AB}}$ is a quantum state (the trace is not preserved in general).

The map $\mathcal{P}_{x}$ is useful for detecting nonclassical correlation because we have the following theorem.

Theorem 1 The equations $\left(I^{\mathrm{A}} \otimes \mathcal{P}_{x}^{\mathrm{B}}\right) \rho^{\mathrm{AB}}=\left(\mathcal{P}_{x}^{\mathrm{A}} \otimes I^{\mathrm{B}}\right) \rho^{\mathrm{AB}}=\rho^{\mathrm{AB}}$ hold if $\rho^{\mathrm{AB}}$ has a product eigenbasis.

Proof. For a bipartite state with a product eigenbasis (PE), $\rho_{\mathrm{PE}}^{\mathrm{AB}}=\sum_{i j} c_{i j}\left|u_{i}\right\rangle^{\mathrm{A}}\left\langle u_{i}\right| \otimes$ $\left|v_{j}\right\rangle^{\mathrm{B}}\left\langle v_{j}\right|$, we have $\operatorname{Tr}_{\mathrm{A}} \rho_{\mathrm{PE}}^{\mathrm{AB}}=\sum_{l}\left(\sum_{k} c_{k l}\right)\left|v_{l}\right\rangle^{\mathrm{B}}\left\langle v_{l}\right|$. Thus

$$
\begin{aligned}
\widetilde{\rho^{\mathrm{AB}}} & \equiv\left(I^{\mathrm{A}} \otimes \Gamma_{x}^{\mathrm{B}}\right) \rho_{\mathrm{PE}}^{\mathrm{AB}}=\sqrt{\left\{\rho_{\mathrm{PE}}^{\mathrm{AB}}\left[I^{\mathrm{A}} \otimes\left(\operatorname{Tr}_{\mathrm{A}} \rho_{\mathrm{PE}}^{\mathrm{AB}}\right)^{x-1}\right]\right\}\{\text { h.c. }\}} \\
& =\sum_{j, f(j) \neq 0} f(j)^{x-1} \sum_{i} c_{i j}\left|u_{i}\right\rangle^{\mathrm{A}}\left\langle u_{i}|\otimes| v_{j}\right\rangle^{\mathrm{B}}\left\langle v_{j}\right|
\end{aligned}
$$

with $f(j)=\sum_{k} c_{k j}$. For this matrix, we have

$$
\operatorname{Tr}_{\mathrm{A}} \widetilde{\rho^{\mathrm{AB}}}=\sum_{t, f(t) \neq 0} f(t)^{x-1} f(t)\left|u_{t}\right\rangle^{\mathrm{B}}\left\langle u_{t}\left|=\sum_{t, f(t) \neq 0} f(t)^{x}\right| u_{t}\right\rangle^{\mathrm{B}}\left\langle u_{t}\right| .
$$

Thus

$$
\begin{aligned}
\widetilde{\widetilde{\mathrm{AB}}} & \equiv\left(I^{\mathrm{A}} \otimes \Gamma_{1 / x}^{\mathrm{B}}\right) \widetilde{\rho^{\mathrm{AB}}}=\sqrt{\left\{\widetilde{\rho^{\mathrm{AB}}}\left[I^{\mathrm{A}} \otimes\left(\operatorname{Tr}_{\mathrm{A}} \widetilde{\rho^{\mathrm{AB}}}\right)^{(1-x) / x}\right]\right\}\{\text { h.c. }\}} \\
& =\sum_{j,} f(j) \neq 0 \\
& f(j)^{x-1} f(j)^{1-x} \sum_{i} c_{i j}\left|u_{i}\right\rangle^{\mathrm{A}}\left\langle u_{i}|\otimes| v_{j}\right\rangle^{\mathrm{B}}\left\langle v_{j}\right| \\
& =\rho^{\mathrm{AB}} .
\end{aligned}
$$

This proves that $\left(I^{\mathrm{A}} \otimes \mathcal{P}_{x}^{\mathrm{B}}\right) \rho_{\mathrm{PE}}^{\mathrm{AB}}=\rho_{\mathrm{PE}}^{\mathrm{AB}}$. It is easy to show that $\left(\mathcal{P}_{x}^{\mathrm{A}} \otimes I^{\mathrm{B}}\right) \rho_{\mathrm{PE}}^{\mathrm{AB}}=\rho_{\mathrm{PE}}^{\mathrm{AB}}$ in the same way.

Here is a simple example to use this map for detecting nonclassical correlation. For the bipartite state $\rho_{0+}$ which has been introduced in (3), $\left(I \otimes \mathcal{P}_{2}\right) \rho_{0+}$ has the eigenvalues (approximately) $0.826,0.375$, and (strictly) 0 (with multiplicity two) which are different from the eigenvalues of $\rho_{0+}, 3 / 4,1 / 4$, and 0 (with multiplicity two), except 0 's. Therefore $\rho_{0+}$ has no product eigenbasis.

\section{Quantification of nonclassical correlation}

For the next step, we define a measure of nonclassical correlation based on the theory of EnCE maps we have seen. Note that, according to the definition, the set of the classically correlated 
states is a nonconvex subset of the set of the separable states. Thus it is not motivating to impose convexity on a measure of nonclassical correlation. We may, however, impose a family of additivity properties [23. In particular, subadditivity is assessed in the following. We begin with non-subadditive measures and later introduce subadditive measures. A strategy to extend the detection ability of subadditive measures is described.

\subsection{Non-subadditive measures}

We first define a non-subadditive measure of nonclassical correlation for a given EnCE map $\Lambda_{\mathrm{EnCE}}$ as follows. Suppose we want to quantify a nonclassical correlation of a bipartite system $\mathrm{AB}$ described by a density matrix $\rho^{\mathrm{AB}}$. Then, we may consider the quantity with subscript $\mathrm{R}$ (L) indicating that the right (left) component is acted by $\Lambda_{\mathrm{EnCE}}$ :

$$
D_{\mathrm{R}, \mathrm{L}}\left(\Lambda_{\mathrm{EnCE}}, \rho^{\mathrm{AB}}\right)=\sum_{s}\left|e_{s}-e^{\prime}{ }_{s}\right|
$$

where $e_{s}$ 's are the eigenvalues of $\rho^{\mathrm{AB}}$ while $e^{\prime} s_{s}$ 's are those of $\left(I^{\mathrm{A}} \otimes \Lambda_{\mathrm{EnCE}}^{\mathrm{B}}\right) \rho^{\mathrm{AB}}$ for "R" $\left[\left(\Lambda_{\mathrm{EnCE}}^{\mathrm{A}} \otimes I^{\mathrm{B}}\right) \rho^{\mathrm{AB}}\right.$ for "L" $] ; e_{s}$ 's and $e^{\prime}{ }_{s}$ 's are aligned, say, in the descending order. We may use the transposition $\Lambda_{\mathrm{T}}$ or the map $\mathcal{P}_{x}$ defined in Definition 7 for $\Lambda_{\mathrm{EnCE}}$. It is obvious that $D$ vanishes if $\rho^{\mathrm{AB}}$ has a product eigenbasis.

We can easily calculate $D_{\mathrm{R}, \mathrm{L}}\left(\Lambda_{\mathrm{EnCE}}, \rho^{\mathrm{AB}}\right)$. For example, it is easy to calculate $D_{\mathrm{R}}\left(\Lambda_{\mathrm{T}}, \rho_{\mathrm{ps}}\right)=$ $2 p$ for the two-qubit state $\rho_{\mathrm{ps}}$ defined in (2).

Another simple example is the quantification of a nonclassical correlation for the bipartite state $\rho_{0+}$ which has been introduced in (3). The eigenvalues of $\rho_{0+}$ are $3 / 4,1 / 4$, and 0 (with multiplicity two). The quantity $D_{\mathrm{R}}\left(\Lambda_{\mathrm{T}}, \rho_{0+}\right)$ vanishes because $\left(I \otimes \Lambda_{\mathrm{T}}\right) \rho_{0+}=\rho_{0+}$. In contrast, $D_{\mathrm{R}}\left(\mathcal{P}_{2}, \rho_{0+}\right)$ does not vanish: as we have computed in an example in the previous section, $\left(I \otimes \mathcal{P}_{2}\right) \rho_{0+}$ has the eigenvalues (approximately) $0.826,0.375$, and (strictly) 0 (with multiplicity two). Thus $D_{\mathrm{R}}\left(\mathcal{P}_{2}, \rho_{0+}\right) \simeq 0.201$.

The last example is to clarify that $D_{\mathrm{R}, \mathrm{L}}$ is not subadditive. For $|\psi\rangle=(|00\rangle+|11\rangle) / \sqrt{2}$, $\left(I^{\mathrm{A}} \otimes \Lambda_{\mathrm{T}}^{\mathrm{B}}\right)|\psi\rangle^{\mathrm{AB}}\langle\psi|$ has the nondegenerate eigenvalue $-1 / 2$ and the degenerate eigenvalue $1 / 2$ with multiplicity three. The eigenvalues of $|\psi\rangle\langle\psi|$ are 1 and 0 with multiplicity three. Thus $D_{\mathrm{R}}\left(\Lambda_{\mathrm{T}},|\psi\rangle\langle\psi|\right)=2$. It is now easy to find the eigenvalues of $\left(I^{\mathrm{AC}} \otimes \Lambda_{\mathrm{T}}^{\mathrm{BD}}\right)|\psi\rangle^{\mathrm{AB}}\langle\psi| \otimes$ $|\psi\rangle^{\mathrm{CD}}\langle\psi|$, which are $-1 / 4$ with multiplicity six and $1 / 4$ with multiplicity ten. This results in $D_{\mathrm{R}}\left(\Lambda_{\mathrm{T}},|\psi\rangle\langle\psi|\otimes| \psi\rangle\langle\psi|\right)=9 / 2$. This value is larger than $2 \times D_{\mathrm{R}}\left(\Lambda_{\mathrm{T}},|\psi\rangle\langle\psi|\right)=4$, indicating that subadditivity does not hold.

\subsection{Subadditive measures}

It has been shown that the measures $D_{\mathrm{R}, \mathrm{L}}$ introduced above are neither additive nor subadditive. Additive or subadditive measures are desirable if one needs to compare systems with different dimensions. Here, subadditive measures are introduced. Let us formally begin with the definition of subadditivity $[23$.

Definition 8 Let $F\left(\rho^{\mathrm{AB}}\right)_{\mathrm{A} \mid \mathrm{B}}$ be a measure of correlation between subsystems $A$ and $B$ of $a$ bipartite system $A B$, where $\mathrm{A} \mid \mathrm{B}$ denotes splitting between $A$ and $B$. Then, $F\left(\rho^{\mathrm{AB}}\right)_{\mathrm{A} \mid \mathrm{B}}$ is called a subadditive measure if and only if the relation $F\left(\rho^{\mathrm{AB}} \otimes \sigma^{\mathrm{CD}}\right)_{\mathrm{AC} \mid \mathrm{BD}} \leq F\left(\rho^{\mathrm{AB}}\right)_{\mathrm{A} \mid \mathrm{B}}+F\left(\sigma^{\mathrm{CD}}\right)_{\mathrm{C} \mid \mathrm{D}}$ holds for density matrices $\rho^{\mathrm{AB}}$ and $\sigma^{\mathrm{CD}}$ of systems $\mathrm{AB}$ and $\mathrm{CD}$ in general.

$\bar{b}$ The calculation is as follows. $D_{\mathrm{R}}\left(\Lambda_{\mathrm{T}}, \rho_{\mathrm{ps}}\right)=[(1+3 p) / 4-(1+p) / 4]+2 \times[(1+p) / 4-(1-p) / 4]+[(1-$ p) $/ 4-(1-3 p) / 4]=2 p$. 
We find that the following quantities $Q_{\mathrm{R}}$ and $Q_{\mathrm{L}}$ satisfy the subadditivity condition if we choose the map $\Lambda_{\text {EnCE }}$ properly. We define them as

$$
Q_{\mathrm{R}, \mathrm{L}}\left(\Lambda_{\mathrm{EnCE}}, \rho^{\mathrm{AB}}\right)=-\log _{2}\left(\frac{1}{N} \sum_{s} \sqrt{e_{s} \widetilde{e_{s}}}\right)
$$

where $e_{s}$ 's are the eigenvalues of $\rho^{\mathrm{AB}}$ and $\widetilde{e_{s}}$ 's are the absolute values of the eigenvalues of $\left(I^{\mathrm{A}} \otimes \Lambda_{\mathrm{EnCE}}^{\mathrm{B}}\right) \rho^{\mathrm{AB}}$ for "R" [ $\left(\Lambda_{\mathrm{EnCE}}^{\mathrm{A}} \otimes I^{\mathrm{B}}\right) \rho^{\mathrm{AB}}$ for "L"]; $e_{s}$ 's and $\widetilde{e_{s}}$ 's are both sorted, say, in descending order; $N=\sqrt{\sum_{s} \widetilde{e_{s}}}$ is a normalization factor which guarantees $Q_{\mathrm{R}, \mathrm{L}} \geq 0$. The measures $Q_{\mathrm{R}, \mathrm{L}}$ vanish if $\left\{e_{s}\right\}=\left\{\widetilde{e_{s}} / N^{2}\right\}$. As for subadditivity, we can prove the following proposition.

Proposition 4 The measure $Q_{\mathrm{R}}\left(\Lambda_{\mathrm{EnCE}}, \rho^{\mathrm{AB}}\right)$ is subadditive if the set of the absolute values of the eigenvalues of $\left(I^{\mathrm{AC}} \otimes \Lambda_{\mathrm{EnCE}}^{\mathrm{BD}}\right)\left(\rho^{\mathrm{AB}} \otimes \sigma^{\mathrm{CD}}\right)$ is given by $\left\{\widetilde{a_{j}} \widetilde{b_{k}}\right\}_{j k}$ where $\widetilde{a_{j}}$ and $\widetilde{b_{k}}$ are the absolute values of the eigenvalues of $\left(I^{\mathrm{A}} \otimes \Lambda_{\mathrm{EnCE}}^{\mathrm{B}}\right) \rho^{\mathrm{AB}}$ and those of $\left(I^{\mathrm{C}} \otimes \Lambda_{\mathrm{EnCE}}^{\mathrm{D}}\right) \sigma^{\mathrm{CD}}$, respectively.

Proof. The proof consists of two steps (i) and (ii).

(i) Consider the two sequences $\left\{p_{i}\right\}_{i=1}^{d}$ and $\left\{q_{i}\right\}_{i=1}^{d}$ of nonnegative real numbers $p_{i}$ and $q_{i}$. Suppose they are sorted: $p_{1} \geq p_{2} \geq \cdots \geq p_{d}$ and $q_{1} \geq q_{2} \geq \cdots \geq q_{d}$. Then, the fidelity $\sum_{i=1}^{d} \sqrt{p_{i} q_{i}}$ for these sorted sequences is larger than that for any two unsorted sequences whose entries are $p_{i}$ 's and $q_{i}$ 's, respectively. This is because, for real numbers $a_{1}, a_{2}, b_{1}$, and $b_{2}$ such that $a_{1} \geq a_{2}$ and $b_{1} \geq b_{2}$, the relation $a_{1} b_{1}+a_{2} b_{2} \geq a_{1} b_{2}+a_{2} b_{1}$ holds.

(ii) Let us write the eigenvalues of $\rho^{\mathrm{AB}}$ as $a_{j}$ and those of $\sigma^{\mathrm{CD}}$ as $b_{k}$. The fidelity $F^{\prime}=$ $\sum_{j=1}^{d^{\mathrm{A}}} \sum_{k=1}^{d^{\mathrm{B}}} \sqrt{\left(a_{j} b_{k}\right)\left(\widetilde{a_{j}} \widetilde{b_{k}}\right)} / N$ with $N=\sqrt{\sum_{j k} \widetilde{a_{j}} \widetilde{b_{k}}}$ involves two possibly unsorted sequences $\left\{\left(a_{j} b_{k}\right)\right\}$ and $\left\{\left(\widetilde{a_{j}} \widetilde{b_{k}}\right)\right\}$; these are unsorted in general even when $\left\{a_{j}\right\},\left\{b_{k}\right\},\left\{\widetilde{a_{j}}\right\}$, and $\left\{\widetilde{b_{k}}\right\}$ are individually sorted. Let us write the fidelity after sorting $\left\{\left(a_{j} b_{k}\right)\right\}$ and $\left\{\left(\widetilde{a_{j}} \widetilde{b_{k}}\right)\right\}$ as $F$. Then, $F^{\prime} \leq F \leq 1$ holds according to the fact (i). Therefore, $0 \leq-\log _{2} F \leq-\log _{2} F^{\prime}=$ $-\log _{2} F_{a}-\log _{2} F_{b}$ holds with $F_{a}=\sum_{j} \sqrt{a_{j} \widetilde{a_{j}}} / \sqrt{\sum_{j} \widetilde{a_{j}}}$ and $F_{b}=\sum_{k} \sqrt{b_{k} \widetilde{b_{k}}} / \sqrt{\sum_{k} \widetilde{b_{k}}}$, where $\left\{a_{j}\right\},\left\{b_{k}\right\},\left\{\tilde{a_{j}}\right\}$, and $\left\{\tilde{b_{k}}\right\}$ are individually sorted.

It is trivial to find a similar condition for $Q_{\mathrm{L}}\left(\Lambda_{\mathrm{EnCE}}, \rho^{\mathrm{AB}}\right)$ to be subadditive. In addition, it is clear that $Q_{\mathrm{R}, \mathrm{L}}$ vanish if $\rho^{\mathrm{AB}}$ has a product eigenbasis. These measures are a sort of logarithmic fidelity and are reminiscent of logarithmic negativity [24, 25, 26]. We find that choosing $\Lambda_{\mathrm{EnCE}}$ from the maps $\Lambda_{\mathrm{T}}$ and $\mathcal{P}_{x}$ introduced in the previous section satisfies the condition of Proposition 4 as we prove below. As for other additivity properties, $Q_{\mathrm{R}, \mathrm{L}}$ is not additive or weakly additive in general owing to sortings of the eigenvalues. This is clear from the following example: For the state $\rho_{\mathrm{ps}}$ defined in (2), with $p$ set to $1 / 3$, we have $Q_{\mathrm{R}}\left(\Lambda_{\mathrm{T}}, \rho_{\mathrm{ps}, p=1 / 3}^{\mathrm{AB}} \otimes \rho_{\mathrm{ps}, p=1 / 3}^{\mathrm{CD}}\right)_{\mathrm{AC} \mid \mathrm{BD}}=-\log _{2}(5 / 18+\sqrt{3} / 3) \simeq 0.226$; this is less than $2 \times Q_{\mathrm{R}}\left(\Lambda_{\mathrm{T}}, \rho_{\mathrm{ps}, p=1 / 3}^{\mathrm{AB}}\right)_{\mathrm{A} \mid \mathrm{B}}=-2 \log _{2}(\sqrt{6} / 6+\sqrt{2} / 3) \simeq 0.370$.

As we have mentioned above, one map that makes the measure $Q_{\mathrm{R}, \mathrm{L}}$ subadditive is the transposition $\Lambda_{\mathrm{T}}$. The subadditivity is easily verified according to the fact that $I^{\mathrm{AC}} \otimes \Lambda_{\mathrm{T}}^{\mathrm{BD}}=$ $\left(I^{\mathrm{A}} \otimes \Lambda_{\mathrm{T}}^{\mathrm{B}}\right)\left(I^{\mathrm{C}} \otimes \Lambda_{\mathrm{T}}^{\mathrm{D}}\right)$. The condition on the set of eigenvalues stated in Proposition 4 is obviously satisfied. In addition, the measures $Q_{\mathrm{R}, \mathrm{L}}\left(\Lambda_{\mathrm{T}}, \rho^{\mathrm{AB}}\right)$ are invariant under local unitary operations. Its invariance under local unitary operations (say, $\left.U^{\mathrm{B}}\right)$ follows from $\left(I^{\mathrm{A}} \otimes \Lambda_{\mathrm{T}}^{\mathrm{B}}\right)\left(I^{\mathrm{A}} \otimes\right.$ $\left.U^{\mathrm{B}} \rho^{\mathrm{AB}} I^{\mathrm{A}} \otimes U^{\dagger^{\mathrm{B}}}\right)=\left(I^{\mathrm{A}} \otimes U^{* \mathrm{~B}}\right)\left(I^{\mathrm{A}} \otimes \Lambda_{\mathrm{T}}^{\mathrm{B}} \rho^{\mathrm{AB}}\right)\left(I^{\mathrm{A}} \otimes U^{* \dagger^{\mathrm{B}}}\right)$. 
It is also easy to find that $\mathcal{P}_{x}$, the map defined in Definition 7, makes $Q_{\mathrm{R}, \mathrm{L}}$ subadditive. Because $\operatorname{Tr}_{\mathrm{AC}} \rho^{\mathrm{AB}} \otimes \rho^{\mathrm{CD}}=\left(\operatorname{Tr}_{\mathrm{A}} \rho^{\mathrm{AB}}\right) \otimes\left(\operatorname{Tr}_{\mathrm{C}} \rho^{\mathrm{CD}}\right)$, we have

$$
\begin{aligned}
& \left(I^{\mathrm{AC}} \otimes \Gamma_{x}^{\mathrm{BD}}\right)\left(\rho^{\mathrm{AB}} \otimes \sigma^{\mathrm{CD}}\right) \\
& =\sqrt{\left\{\rho^{\mathrm{AB}}\left[I^{\mathrm{A}} \otimes\left(\operatorname{Tr}_{\mathrm{A}} \rho^{\mathrm{AB}}\right)^{x-1}\right] \otimes \sigma^{\mathrm{CD}}\left[I^{\mathrm{C}} \otimes\left(\operatorname{Tr}_{\mathrm{C}} \sigma^{\mathrm{CD}}\right)^{x-1}\right]\right\}\{\text { h.c. }\}} \\
& =\left(I^{\mathrm{A}} \otimes \Gamma_{x}^{\mathrm{B}}\right) \rho^{\mathrm{AB}} \otimes\left(I^{\mathrm{C}} \otimes \Gamma_{x}^{\mathrm{D}}\right) \sigma^{\mathrm{CD}} .
\end{aligned}
$$

Hence, $\left(I^{\mathrm{AC}} \otimes \mathcal{P}^{\mathrm{BD}}\right)\left(\rho^{\mathrm{AB}} \otimes \sigma^{\mathrm{CD}}\right)=\left(I^{\mathrm{A}} \otimes \mathcal{P}_{x}^{\mathrm{B}}\right) \rho^{\mathrm{AB}} \otimes\left(I^{\mathrm{C}} \otimes \mathcal{P}_{x}^{\mathrm{D}}\right) \sigma^{\mathrm{CD}}$ holds. Therefore, by Proposition 4, we find that $Q_{\mathrm{R}}\left(\mathcal{P}_{x}, \rho^{\mathrm{AB}}\right)$ is subadditive [and $Q_{\mathrm{L}}\left(\mathcal{P}_{x}, \rho^{\mathrm{AB}}\right)$ either]. In addition, it is invariant under local unitary operations because $I^{\mathrm{A}, \mathrm{B}} \otimes \Gamma_{x}^{\mathrm{B}, \mathrm{A}}$ commutes with a local unitary transformation, by its definition.

The problem is that $Q_{\mathrm{R}}$ and $Q_{\mathrm{L}}$ are different in general. To solve this problem, we suggest using the average

$$
\widetilde{Q}\left(\Lambda_{\mathrm{EnCE}}, \rho^{\mathrm{AB}}\right)=\frac{Q_{\mathrm{R}}\left(\Lambda_{\mathrm{EnCE}}, \rho^{\mathrm{AB}}\right)+Q_{\mathrm{L}}\left(\Lambda_{\mathrm{EnCE}}, \rho^{\mathrm{AB}}\right)}{2} .
$$

This becomes subadditive and invariant under local unitary operations if both $Q_{\mathrm{R}}$ and $Q_{\mathrm{L}}$ are subadditive and invariant under local unitary operations. It is easy to find that $\Lambda_{\mathrm{T}}$ and $\mathcal{P}_{x}$ are both useful for this purpose.

\subsection{Computational complexity}

An advantage of using the measure $Q_{\mathrm{R}, \mathrm{L}}$ defined in (4) and the measure $\tilde{Q}$ defined in (5) is their relatively small computational cost when $\Lambda_{\mathrm{T}}$ or $\mathcal{P}_{x}$ is chosen for $\Lambda_{\mathrm{EnCE}}$.

Consider a bipartite system with the dimension $d^{\mathrm{A}}\left(d^{\mathrm{B}}\right)$ of the Hilbert space of its subsystem A (B). For a density matrix $\rho^{\mathrm{AB}},\left(I \otimes \Lambda_{\mathrm{T}}\right) \rho^{\mathrm{AB}}$ is computed with $O\left(d^{\mathrm{A}^{2}} d^{\mathrm{B}^{2}}\right)$ basic floating-point operations. This is less than the cost of diagonalization of $\left(I \otimes \Lambda_{\mathrm{T}}\right) \rho^{\mathrm{AB}}$, which takes $O\left(d^{\mathrm{A}^{3}} d^{\mathrm{B}^{3}}\right)$ basic floating-point operations. The computation of $\left(I \otimes \mathcal{P}_{x}\right) \rho^{\mathrm{AB}}$ is a little expensive because it involves a square root of a matrix. The cost of computing $\left(I \otimes \mathcal{P}_{x}\right) \rho^{\mathrm{AB}}$ is $O\left(d^{\mathrm{A}^{3}} d^{\mathrm{B}^{3}}\right)$, same as the cost of diagonalizing $\left(I \otimes \mathcal{P}_{x}\right) \rho^{\mathrm{AB}}$.

Once the eigenvalues of $\left(I \otimes \Lambda_{\mathrm{EnCE}}\right) \rho^{\mathrm{AB}}$ is computed, it takes only $O\left(d^{\mathrm{A}} d^{\mathrm{B}}\right)$ basic floatingpoint operations to compute $Q_{\mathrm{R}, \mathrm{L}}$ and $\tilde{Q}$. Therefore, the time complexity of computing these measures is $O\left(d^{\mathrm{A}^{3}} d^{\mathrm{B}^{3}}\right)$ when $\Lambda_{\mathrm{T}}$ or $\mathcal{P}_{x}$ is chosen.

\subsection{Extending the detection range}

One might be curious if $\mathcal{P}_{x}$, the map defined in Definition 7 , is more useful than the transposition $\Lambda_{\mathrm{T}}$ in detecting nonclassical correlation by using the measure $\widetilde{Q}$ defined in (5). First, $\widetilde{Q}\left(\Lambda_{\mathrm{T}}, \rho_{0+}^{\mathrm{AB}}\right)$ vanishes while $\widetilde{Q}\left(\mathcal{P}_{2}, \rho_{0+}^{\mathrm{AB}}\right) \simeq 7.00 \times 10^{-3}$ for the state $\rho_{0+}$ defined in (3). Second, $\widetilde{Q}\left(\Lambda_{\mathrm{T}},|\psi\rangle^{\mathrm{AB}}\langle\psi|\right)=1$ while $\widetilde{Q}\left(\mathcal{P}_{x},|\psi\rangle^{\mathrm{AB}}\langle\psi|\right)$ vanishes for $|\psi\rangle=(|00\rangle+|11\rangle) / \sqrt{2}$. Therefore, generally speaking, $\widetilde{Q}\left(\mathcal{P}_{x}, \rho^{\mathrm{AB}}\right)$ is neither stronger nor weaker than $\widetilde{Q}\left(\Lambda_{\mathrm{T}}, \rho^{\mathrm{AB}}\right)$. One may further claim that $\mathcal{P}_{x}$ is not very useful because it vanishes for the Bell state. Nevertheless, this is not a serious drawback as we have a quick solution as follows.

There is a way to utilize these measures to produce a stronger measure. Suppose we have non-negative, subadditive, and local-unitary-invariant measures $M_{1}, \ldots, M_{N}$. Then, the weighted average $\sum_{k} w_{k} M_{k}$ with $w_{k}>0$ is also a measure which is non-negative, subadditive, and invariant under local unitary operations. It detects nonclassical correlation for the states for which any one of $M_{1}, \ldots, M_{N}$ is nonvanishing. 
Thus we easily produce the stronger measure

$$
w_{\mathrm{T}} \widetilde{Q}\left(\Lambda_{\mathrm{T}}, \rho^{\mathrm{AB}}\right)+\sum_{k} w_{k} \widetilde{Q}\left(\mathcal{P}_{x_{k}}, \rho^{\mathrm{AB}}\right)
$$

with $x_{k} \in \mathbf{R}, x_{k} \neq 1$, and $w_{\mathrm{T}}, w_{k}>0$. This measure does not vanish for $\rho_{0+}$ and $|\psi\rangle\langle\psi|$.

\section{Concluding remarks}

We have seen several different usages of the EnCE map theory. We believe that this theory works as a useful template to detect and quantify nonclassical correlation based on the Oppenheim-Horodecki separation of classical/nonclassical correlations. The EnCE map theory has been constructed in analogy to the PnCP map theory in the present paper. One important difference between these theories is that the class of EnCE maps includes nonlinear EnCE maps. This is because linear EnCE maps are very limited due to the fact that any linear EP map can be decomposed into unitary operations and a transposition (Proposition 2). Nonlinearity of a map is not a significant problem as far as $I \otimes \Lambda_{\mathrm{EnCE}}$ and $\Lambda_{\mathrm{EnCE}} \otimes I$ are defined appropriately for an EnCE map $\Lambda_{\mathrm{EnCE}}$ in the way that $I \otimes \Lambda_{\mathrm{EnCE}}$ and $\Lambda_{\mathrm{EnCE}} \otimes I$ preserve the eigenvalues of any state that has a product eigenbasis. If one intends to rule out nonlinearity, a possible extension of the theory is to go beyond the tacit assumption of the Hermiticity-preserving property of a map. This will be studied in the future.

On the basis of the EnCE map theory, we have defined two subadditive measures, $\widetilde{Q}\left(\Lambda_{\mathrm{T}}, \rho^{\mathrm{AB}}\right)$ and $\widetilde{Q}\left(\mathcal{P}_{x}, \rho^{\mathrm{AB}}\right)$. These are neither stronger nor weaker to each other in the detection range, and not so strong as the measure by Piani et al. [12] that is perfect in the detection range albeit intractable in computational cost. The advantage of our measures is the complexity: they are calculated within polynomial time in the dimension of the Hilbert space. We have shown a way to relax the drawback of the detection range; their weighted average is stronger than themselves and remains subadditive as shown in Section 4.4. A certain optimization over the weights and the choices of $x$ 's will be investigated in future work.

One might be curious about an extension of the measures to multipartite splitting. This is achieved by taking a minimum, maximum, or average of a measure over all possible bipartite splittings of the multipartite system. In considering the possible combinations of subsystems for a bipartite splitting, we should be careful about the fact that having product eigenbases for $\mathrm{A} \mid \mathrm{BC}$ splitting and $\mathrm{AB} \mid \mathrm{C}$ splitting does not imply having a product eigenbasis for $\mathrm{A}|\mathrm{B}| \mathrm{C}$ splitting. A typical example is the state $(|000\rangle\langle 000|+| 1+1\rangle\langle 1+1|) / 2$ with $|+\rangle=(|0\rangle+|1\rangle) / \sqrt{2}$. This state does not have a product eigenbasis for $\mathrm{A}|\mathrm{B}| \mathrm{C}$ splitting while it has for $\mathrm{A} \mid \mathrm{BC}$ and $\mathrm{AB} \mid \mathrm{C}$ splittings. A proper claim is that having product eigenbases for the $\mathrm{A}|\mathrm{BC}, \mathrm{AB}| \mathrm{C}$, and $\mathrm{AC} \mid \mathrm{B}$ splittings implies having a product eigenbasis for $\mathrm{A}|\mathrm{B}| \mathrm{C}$ splitting. More generally, an $m$-partite state $\rho^{1 \ldots m}$ has a fully product eigenbasis if and only if $\rho^{1 \ldots m}$ has a product eigenbasis for every possible bipartite splitting separating $\{1, \ldots, m\}$ into two sets. The proof is given in Appendix 1. In addition, as a different direction to study the measures for a multipartite system, one may seek for a monogamy property, namely, a sort of restriction to a subsystem in the amount of correlation with other subsystems when it has a correlation with a particular subsystem (See, e.g., Ref. [27] and references therein). It is an open problem if a measure in the form of (4) fulfills a certain monogamy property alone or together with a different measure of classical or nonclassical correlation. 
In summary, a comprehensive framework, called the EnCE map theory, to detect and quantify nonclassical correlation of a bipartite system has been proposed. The average logarithmic fidelity $\widetilde{Q}\left(\Lambda_{\mathrm{EnCE}}, \rho^{\mathrm{AB}}\right)$ has been introduced as a subadditive measure for a properly-chosen EnCE map $\Lambda_{\mathrm{EnCE}}$. It is computable within polynomial time in the dimension of the Hilbert space. A simple way to extend the detection range by a collection of measures has been developed.

\section{Acknowledgements}

A.S. and M.N. are supported by the "Open Research Center" Project for Private Universities: matching fund subsidy from MEXT. R.R. is supported by the FIRST program of JSPS. A.S., R.R., and M.N. are supported by Grants-in-Aid for Scientific Research from JSPS (Grant Nos. 21800077, 1907329, and 19540422, respectively).

\section{References}

1. J. Gruska (1999), Quantum Computing, McGraw-Hill (London).

2. M. A. Nielsen and I. L. Chuang (2000), Quantum Computation and Quantum Information, Cambridge University Press (Cambridge).

3. C. H. Bennett, D. P. DiVincenzo, C. A. Fuchs, T. Mor, E. Rains, P. W. Shor, J. A. Smolin, and W. K. Wootters (1999), Quantum nonlocality without entanglement, Phys. Rev. A, 59, pp. 1070-1091.

4. H. Ollivier and W. H. Zurek (2001), Quantum discord: A measure of the quantumness of correlations, Phys. Rev. Lett., 88, pp. 017901-1-4.

5. J. Oppenheim, M. Horodecki, P. Horodecki, and R. Horodecki (2002), Thermodynamical approach to quantifying quantum correlations, Phys. Rev. Lett., 89, pp. 180402-1-4.

6. M. B. Plenio and S. Virmani (2007), An introduction to entanglement measures, Quantum Inf. Comput., Vol. 7, pp. 1-51.

7. A. Datta, A. Shaji, and C. M. Caves (2008), Quantum discord and the power of one qubit, Phys. Rev. Lett., 100, pp. 050502-1-4.

8. E. Knill and R. Laflamme (1998), Power of one bit of quantum information, Phys. Rev. Lett., 81, pp. 5672-5675.

9. M. Horodecki, P. Horodecki, R. Horodecki, J. Oppenheim, A. Sen(De), U. Sen, and B. SynakRadtke (2005), Local versus nonlocal information in quantum-information theory: Formalism and phenomena, Phys. Rev. A, 71, pp. 062307-1-25.

10. B. Groisman, D. Kenigsberg, and T. Mor, "Quantumness" versus "classicality" of quantum states, quant-ph/0703103.

11. A. SaiToh, R. Rahimi, and M. Nakahara (2008), Nonclassical correlation in a multipartite quantum system: Two measures and evaluation, Phys. Rev. A, 77, pp. 052101-1-9.

12. M. Piani, P. Horodecki, and R. Horodecki (2008), No-local-broadcasting theorem for multipartite quantum correlations, Phys. Rev. Lett., 100, pp. 090502-1-4.

13. E. Davies (1978), Information and quantum measurement, IEEE Trans. Inf. Theory, 24, pp. 596599.

14. S. Luo (2008), Using measurement-induced disturbance to characterize correlations as classical or quantum, Phys. Rev. A, 77, pp. 022301-1-5.

15. A. Datta and S. Gharibian (2009), Signatures of nonclassicality in mixed-state quantum computation, Phys. Rev. A, 79, pp. 042325-1-8.

16. A. Peres (1996), Separability criterion for density matrices, Phys. Rev. Lett., 77, pp. 1413-1415.

17. M. Horodecki, P. Horodecki, and R. Horodecki (1996), Separability of mixed states: necessary and sufficient conditions, Phys. Lett. A, 223, pp. 1-8. 
18. P. Horodecki (1997), Separability criterion and inseparable mixed states with positive partial transposition, Phys. Lett. A, 232, pp. 333-339.

19. A. SaiToh, R. Rahimi, and M. Nakahara (2008), Evaluating measures of nonclassical correlation in a multipartite quantum system, Int. J. Quant. Inf., 6 (Supp. 1), pp. 787-793.

20. R. Horodecki, P. Horodecki, M Horodecki, and K. Horodecki (2009), Quantum entanglement, Rev. Mod. Phys., 81, pp. 865-942.

21. E. P. Wigner (1960), Phenomenological distinction between unitary and antiunitary symmetry operators, J. Math. Phys., 1, pp. 414-416.

22. E. P. Wigner (1960), Normal form of antiunitary operators, J. Math. Phys., 1, pp. 409-413.

23. C. H. Bennett, A. W. Harrow, D. W. Leung, and J. A. Smolin (2003), On the capacities of bipartite hamiltonians and unitary gates, IEEE Trans. Inf. Theory, 49, pp. 1895-1911.

24. M. B. Plenio (2005), Logarithmic negativity: A full entanglement monotone that is not convex, Phys. Rev. Lett., 95, pp. 090503-1-4.

25. K. Życzkowski, P. Horodecki, A. Sanpera, and M. Lewenstein (1998), Volume of the set of separable states, Phys. Rev. A, 58, pp. 883-892.

26. G. Vidal and R. F. Werner (2002), Computable measure of entanglement, Phys. Rev. A, 65, pp. 032314-1-11.

27. M. Koashi and A. Winter (2004), Monogamy of quantum entanglement and other correlations, Phys. Rev. A, 69, pp. 022309-1-6.

\section{Appendix A Theorem on multipartite product eigenbasis}

Theorem 2 An m-partite state $\rho^{1 \ldots m}$ has a fully product eigenbasis if and only if $\rho^{1 \ldots m}$ has a product eigenbasis for every possible bipartite splitting separating $\{1, \ldots, m\}$ into two sets.

Proof. It is trivial that $\rho^{1 \ldots m}$ has a product eigenbasis for every possible bipartite splitting if it has a fully product eigenbasis.

Now we prove the converse. By lemma 1 introduced below, the density matrix has a product eigenbasis for the $1|2| 34 \ldots m$ splitting and that for the $12|3| 4 \ldots m$ splitting. The latter fact implies that $\rho^{1 \ldots m}$ 's eigenbasis is a product of the eigenbasis of the reduced density matrix $\rho^{12}$, that of $\rho^{3}$, and that of $\rho^{4 \ldots m}$. The former fact implies that the reduced density matrix $\rho^{12}$ has a product eigenbasis. Therefore, $\rho^{1 \ldots m}$ has a product eigenbasis for the $1|2| 3 \mid 4 \ldots m$ splitting.

Next, we use the fact that $\rho^{1 \ldots m}$ has a product eigenbasis for the $123|4| 56 \ldots m$ splitting by lemma 1. Now it is found that $\rho^{1 \ldots m}$ has a product eigenbasis for the $1|2| 3|4| 56 \ldots m$ splitting.

Using the same logic continuously, the converse is proved.

Lemma 1 A tripartite density matrix $\rho^{\mathrm{ABC}}$ has a tripartite product eigenbasis if and only if it has a bipartite product eigenbasis for each of all the bipartite splittings.

Proof. It is trivial that $\rho^{\mathrm{ABC}}$ has a product eigenbasis for any bipartite splitting if it has a tripartite product eigenbasis.

Now we prove the converse. Having a bipartite product eigenbasis for any bipartite splitting implies that

$$
\begin{aligned}
\rho^{\mathrm{ABC}} & =\sum_{i j} a_{i j}\left|r_{i}\right\rangle^{\mathrm{A}}\left\langle r_{i}|\otimes| s_{j}\right\rangle^{\mathrm{BC}}\left\langle s_{j}\right| \\
& =\sum_{k l} b_{k l}\left|t_{k}\right\rangle^{\mathrm{AB}}\left\langle t_{k}|\otimes| u_{l}\right\rangle^{\mathrm{C}}\left\langle u_{l}\right| \\
& =\sum_{m n} c_{m n}\left|v_{m}\right\rangle^{\mathrm{AC}}\left\langle v_{m}|\otimes| w_{n}\right\rangle^{\mathrm{B}}\left\langle w_{n}\right|,
\end{aligned}
$$

where $\left|r_{i}\right\rangle^{\mathrm{A}},\left|s_{j}\right\rangle^{\mathrm{BC}},\left|t_{k}\right\rangle^{\mathrm{AB}},\left|u_{l}\right\rangle^{\mathrm{C}},\left|v_{m}\right\rangle^{\mathrm{AC}}$, and $\left|w_{n}\right\rangle^{\mathrm{B}}$ are eigenvectors of the reduced density matrices of the indicated subsystems; $a_{i j}, b_{k l}$, and $c_{m n}$ are eigenvalues of $\rho^{\mathrm{ABC}}$. 
14 Mathematical framework for detection ...

This leads to that

(i) An eigenbasis of $\rho^{\mathrm{ABC}}$ is a product of an eigenbasis of $\operatorname{Tr}_{\mathrm{BC}} \rho^{\mathrm{ABC}}$ and that of $\operatorname{Tr}_{\mathrm{A}} \rho^{\mathrm{ABC}}$.

(ii) Matrix $\operatorname{Tr}_{\mathrm{A}} \rho^{\mathrm{ABC}}$ is represented as

$$
\begin{aligned}
\operatorname{Tr}_{\mathrm{A}} \rho^{\mathrm{ABC}} & =\sum_{k l} b_{k l} \sigma_{k}^{\mathrm{B}} \otimes\left|u_{l}\right\rangle^{\mathrm{C}}\left\langle u_{l}\right| \\
& =\sum_{m n} c_{m n}\left|w_{n}\right\rangle^{\mathrm{B}}\left\langle w_{n}\right| \otimes \sigma_{m}^{\prime \mathrm{C}}
\end{aligned}
$$

with $\sigma_{k}^{\mathrm{B}}=\operatorname{Tr}_{\mathrm{A}}\left|t_{k}\right\rangle^{\mathrm{AB}}\left\langle t_{k}\right|$ and ${\sigma^{\prime}}_{m}^{\mathrm{C}}=\operatorname{Tr}_{\mathrm{A}}\left|v_{m}\right\rangle^{\mathrm{AC}}\left\langle v_{m}\right|$.

From (ii), we find that

$$
\begin{aligned}
\operatorname{Tr}_{\mathrm{A}} \rho^{\mathrm{ABC}}\left|w_{x}\right\rangle^{\mathrm{B}}\left|u_{y}\right\rangle^{\mathrm{C}} & =\left(\sum_{k} b_{k y} \sigma_{k}^{\mathrm{B}}\left|w_{x}\right\rangle^{\mathrm{B}}\right)\left|u_{y}\right\rangle^{\mathrm{C}} \\
& =\left|w_{x}\right\rangle^{\mathrm{B}}\left(\sum_{m} c_{m x} \sigma_{m}^{\prime}\left|u_{y}\right\rangle^{\mathrm{C}}\right) .
\end{aligned}
$$

This implies that $\left(\sum_{k} b_{k y} \sigma_{k}^{\mathrm{B}}\left|w_{x}\right\rangle^{\mathrm{B}}\right)=p_{x y}\left|w_{x}\right\rangle^{\mathrm{B}}$ with $p_{x y}={ }^{\mathrm{C}}\left\langle u_{y}\left|\sum_{m} c_{m x} \sigma_{m}^{\prime \mathrm{C}}\right| u_{y}\right\rangle^{\mathrm{C}}$. Hence $\left\{\left|w_{x}\right\rangle^{\mathrm{B}}\left|u_{y}\right\rangle^{\mathrm{C}}\right\}$ is an eigenbasis of $\operatorname{Tr}_{\mathrm{A}} \rho^{\mathrm{ABC}}$. This fact and (i) complete the proof. 